\title{
Exact Single Traveling Wave Solutions for Generalized Fractional Gardner Equations
}

\author{
Zhao Li $\mathbb{D}^{1}{ }^{1}$ Tianyong Han, ${ }^{1}$ and Chun Huang ${ }^{2}$ \\ ${ }^{1}$ College of Computer Science, Chengdu University, Chengdu 610106, China \\ ${ }^{2}$ Faculty of Education, Sichuan Vocational and Technical College, Suining 629000, China \\ Correspondence should be addressed to Zhao Li; lizhao10.26@163.com
}

Received 26 August 2020; Revised 9 November 2020; Accepted 25 November 2020; Published 7 December 2020

Academic Editor: Maria Patrizia Pera

Copyright (C) 2020 Zhao Li et al. This is an open access article distributed under the Creative Commons Attribution License, which permits unrestricted use, distribution, and reproduction in any medium, provided the original work is properly cited.

In this paper, the classification of all single traveling wave solutions to generalized fractional Gardner equations is presented by utilizing the complete discrimination system method. Under the fractional traveling wave transformation, generalized fractional Gardner equations can be reduced to an ordinary differential equations. All possible exact traveling wave solutions are given through the complete discrimination system of the fourth-order polynomial. Moreover, graphical representations of different kinds of the exact solutions reveal that the method is of significance for searching the exact solutions to generalized fractional Gardner equations.

\section{Introduction}

It is common knowledge that fractional partial differential equations (FPDEs) [1-9] have gained great attention because they have been widely used to model various complex physical phenomena in the domain of science and engineering. Therefore, it is of great significance to search the exact traveling wave solutions [10-24] of FPDEs in the research of nonlinear science, which can accurately reflect the propagation of nonlinear waves and better understand nonlinear physical phenomena. So far, many powerful methods have been established and developed to analyze the exact solutions to the FPDE, which include the $\left(G^{\prime} / G\right)$-expansion method $[25,26]$, the integral bifurcations $[27,28]$, the Lie symmetry analysis method $[29,30]$, first integral method [31], modified trial equation method [32], the exp-function method [33], F-expansion method [34], and the Kudryashov method [35].

In this paper, we shall consider the following generalized fractional Gardner equation [36]:

$$
\begin{gathered}
D_{t}^{\alpha} u+p u_{x}+\left(q u^{n}+r u^{2 n}\right) u_{x}+u_{x x x}=0, \\
n \geq 0, r<0,0<\alpha \leq 1,
\end{gathered}
$$

where $D_{t}^{\alpha} u$ is the conformable derivative of $u$ depending on the variable $t . u(t, x)$ represents the amplitude of the wave mode, and variables $t$ and $x$ represent the time and spatial variable, respectively. The coefficients $p, q$, and $r$ are constants. Equation (1) can be usually used to describe the nonlinear propagation of ion-acoustic waves at an unmagnetized plasma. As we all know, equation (1) is a kind of very important FPDE. When the parameters of equation (1) are changed, it can be simplified to the following famous nonlinear FPDE. For example, when $n=1, q \neq 0$, and $r=0$, equation (1) becomes the fractional $\mathrm{KdV}$ equation; when $n=1, q=0$, and $r \neq 0$, equation (1) becomes the fractional mKdV equation; and when $n=1, q \neq 0$, and $r \neq 0$, equation (1) becomes a fractional $\mathrm{KdV}-\mathrm{mKdV}$ equation. In [36], Reazadeh et al. obtained the hyperbolic and trigonometric function solutions to the generalized fractional Gardner equation by using modified Kudryashov method and hyperbolic function method, respectively. But, their research only focused on acquiring the hyperbolic and trigonometric function solutions. Motivated by the aforementioned discussion, in the paper, we will construct new exact traveling wave solution to the generalized fractional Gardner equations via the polynomial method. 
The complete discrimination system for the polynomial method was first proposed by Liu [37]. It is one of the most powerful methods to find the single traveling wave solutions to partial differential equations (PDEs). With the development of fractional calculus, the study of exact solution to FPDEs has been gaining more and more attention by many experts and scholars. Because of the complexity of fractional derivative, the exact solution of FPDE develops very slowly than PDE of integers. Many scholars [38, 39] have been trying to find new methods to construct the exact solutions of FPDEs. Recently, Khalil et al. [40] introduced the conformable fractional derivative. FPDEs can be reduced into nonlinear ordinary differential equations by the fractional traveling wave transformation. In the paper, we will find the exact solutions to the generalized fractional Gardner equation by the complete discrimination system of the polynomial method.

The main objective of the paper is to draw support from the complete discrimination system to construct exact traveling wave solutions to the generalized fractional Gardner equation. In Section 2, we review the definition of conformable derivative and introduce the complete discrimination system for constructing the exact traveling wave solutions of FPDE. Then, in Section 3, we discuss the exact solutions to the generalized fractional Gardner equation by using the complete discrimination system. Finally, we give a brief conclusion in Section 4.

\section{Mathematical Preliminaries}

2.1. The Conformable Derivative. The definition and properties of the conformable derivative are defined as

Definition 1. Let $f:[0, \infty) \longrightarrow \mathbf{R}$. Then, the conformable derivative of $f$ of order $\alpha$ is defined as

$$
\begin{array}{r}
D_{t}^{\alpha} f(t)=\lim _{\varepsilon \longrightarrow 0} \frac{f\left(t+\varepsilon t^{1-\alpha}\right)-f(t)}{\varepsilon}, \\
\forall t \in(0,+\infty), \alpha \in(0,1],
\end{array}
$$

and the function $f$ is $\alpha$-conformable differentiable at a point $t$ if the limit in equation (2) exists.

Remark 1. The conformable derivative possesses many important properties (See $[41,42]$ and references therein). In recent years, several scholars [43-47] have developed and constructed exact solutions of FPDE in the sense of conformable derivative.

2.2. Description of the Method. Consider the following conformable FPDE:

$$
P\left(u, D_{t}^{\alpha} u, u_{x}, D_{t}^{2 \alpha} u, u_{x x}, u_{x x x}, \ldots\right)=0, \quad 0<\alpha \leq 1,
$$

where $u=u(t, x)$ is an unknown function.

Introducing the following fractional traveling wave transformation,

$$
\begin{aligned}
u(t, x) & =u(\xi), \\
\xi & =k\left(x-\frac{\omega t^{\alpha}}{\alpha}\right),
\end{aligned}
$$

where $k$ and $\omega$ are nonzero constants.

Substituting equation (4) into equation (3), then equation (3) can be converted into the following integer-order ordinary differential equation:

$$
Q\left(u, u^{\prime}, u^{\prime \prime}, \ldots\right)=0
$$

where $Q$ is a polynomial in $u$ and its derivatives and notation $\left(^{\prime}\right)$ is the derivative with respect to $\xi$.

Equation (5) is usually reduced to

$$
\left(u^{\prime}\right)^{2}=F(u)
$$

where $F(u)$ is a polynomial.

Then, integrating equation (6) once, we can obtain

$$
\pm\left(\xi-\xi_{0}\right)=\int \frac{\mathrm{d} u}{\sqrt{F(u)}}
$$

where $\xi_{0}$ is an integral constant.

According to the above procedures, recent results have been reported via the complete discrimination system [48-50].

\section{Applications}

When $n=1, q \neq 0$, and $r \neq 0$, equation (1) becomes the following fractional $\mathrm{KdV}-\mathrm{mKdv}$ equation:

$$
D_{t}^{\alpha} u+p u_{x}+\left(q u+r u^{2}\right) u_{x}+u_{x x x}=0 .
$$

Substituting equation (4) into equation (8), equation (8) can be converted to an ordinary differential equation as follows:

$$
-k \omega u^{\prime}+p k u^{\prime}+\left(q u+r u^{2}\right) k u^{\prime}+k^{3} u^{\prime \prime \prime}=0 .
$$

Integrating the above once with respect to $\xi$, we obtain

$$
k^{3} u^{\prime \prime}+(p-\omega) k u+\frac{k q}{2} u^{2}+\frac{k r}{3} u^{3}=c_{0} .
$$

Multiplying equation (9) on both sides by $u^{\prime}$ and then integrating it with respect to $\xi$ again,

$$
k^{3}\left(u^{\prime}\right)^{2}+\frac{(p-\omega) k}{2} u^{2}+\frac{k q}{6} u^{3}+\frac{k r}{12} u^{4}=c_{0} u+c_{1},
$$

where $c_{0}$ and $c_{1}$ are integral constants. Then, we obtain

$$
\left(u^{\prime}\right)^{2}=-\frac{r}{12 k^{2}} u^{4}-\frac{q}{6 k^{2}} u^{3}-\frac{(p-\omega)}{2 k^{2}} u^{2}+\frac{c_{0}}{k^{3}} u+\frac{c_{1}}{k^{3}} .
$$

Suppose that $a_{4}=-r / 12 k^{2}, a_{3}=-q / 6 k^{2}, a_{2}=-p-$ $\omega / 2 k^{2}, a_{1}=c_{0} / k^{3}$, and $a_{0}=c_{1} / k^{3}$; then,

$$
\left(u^{\prime}\right)^{2}=a_{4} u^{4}+a_{3} u^{3}+a_{2} u^{2}+a_{1} u+a_{0} .
$$

Making the transformation, we obtain 


$$
\begin{aligned}
& \Phi=\left(a_{4}\right)^{1 / 4}\left(u+\frac{a_{3}}{4 a_{4}}\right), \\
& \xi_{1}=\left(a_{4}\right)^{1 / 4} \xi .
\end{aligned}
$$

Substituting transformation (14) into equation (13), it will be changed into

$$
\Phi_{\xi_{1}}^{2}=\Phi^{4}+b_{1} \Phi^{2}+b_{2} \Phi+b_{3}
$$

where $b_{1}=a_{2} / \sqrt{a_{4}}, b_{2}=\left(a_{3}^{3} / 8 a_{4}^{2}-a_{2} a_{3} / 2 a_{4}+a_{1}\right)\left(a_{4}\right)^{-1 / 4}$, and $b_{3}=-3 a_{3}^{4} / 256 a_{4}^{3}+a_{2} a_{3}^{2} / 16 a_{4}^{2}-a_{1} a_{3} / 4 a_{4}+a_{0}$.

Integrating equation (15) once, we obtain

$$
\pm\left(\xi_{1}-\xi_{0}\right)=\int \frac{\mathrm{d} \Phi}{\sqrt{\Phi^{4}+b_{1} \Phi^{2}+b_{2} \Phi+b_{3}}},
$$

where $\xi_{0}$ is the integration constant.

Let $G(\Phi)=\Phi^{4}+b_{1} \Phi^{2}+b_{2} \Phi+b_{3}$; then, we can obtain its complete discrimination system:

$$
\begin{aligned}
& D_{1}=4, \\
& D_{2}=-b_{1}, \\
& D_{3}=-2 b_{1}^{3}+8 b_{1} b_{3}-9 b_{2}^{2}, \\
& E_{2}=9 b_{1}^{2}-32 b_{1} b_{3}, \\
& D_{4}=-b_{1}^{3} b_{2}^{2}+4 b_{1}^{4} b_{3}+36 b_{1} b_{2}^{2} b_{3}-32 b_{1}^{2} b_{3}^{2}-\frac{27}{4} b_{2}^{4}+64 b_{3}^{3} .
\end{aligned}
$$

Integrating formula (16), we will obtain the exact traveling wave solutions of equation (8) under nine cases.

Case 1. $D_{2}<0, D_{3}=0$, and $D_{4}=0 . G(\Phi)$ has a pair of conjugate complex roots of multiplicities two, i.e.,

$$
G(\Phi)=\left[(\Phi-\beta)^{2}+\gamma^{2}\right]^{2},
$$

where $\gamma>0$. By using equation (16), we attain

$$
\xi_{1}-\xi_{0}=\int \frac{\mathrm{d} \Phi}{(\Phi-\beta)^{2}+\gamma^{2}}=\frac{1}{\gamma} \arctan \frac{\Phi-\beta}{\gamma} .
$$

Then, equation (19) is simplified to

$$
\Phi\left(\xi_{1}\right)=\gamma \tan \left(\gamma\left(\xi_{1}-\xi_{0}\right)\right)+\beta,
$$

which is a trigonometric function solution. Namely, when $b_{1}>0, b_{2}=0$, and $b_{3}=b_{1}^{2} / 4$; therefore, $\gamma=b_{1} / 2$ and then the solution of equation (15) is expressed as follows:

$$
u_{1}(\xi)= \pm a_{4}^{-1 / 4} \gamma \tan \left(\gamma\left(a_{4}^{1 / 4} \xi-\xi_{0}\right)\right)-\frac{a_{3}}{4 a_{4}} .
$$

For instance, when $k=1, p=2, \omega=4, q=-12, r=-12$, $c_{0}=0, c_{1}=11 / 16$, and $\xi_{0}=0$, we can obtain a trigonometric solution of equation (8) as follows. Under the given parameters, we draw the traveling wave $3 \mathrm{D}$ solution surfaces and corresponding 2D solution graphs for the obtained solution $u_{1}(t, x)$ in Figures 1 and 2:

$$
u_{1}(t, x)=\frac{1}{2} \tan \left(\frac{1}{2} x-\frac{2 t^{\alpha}}{\alpha}\right)-\frac{1}{2} .
$$

Case 2. $D_{2}=0, D_{3}=0$, and $D_{4}=0 . G(\Phi)$ has real roots of multiplicities four, namely,

$$
G(\Phi)=\Phi^{4}
$$

By using equation (16), we can obtain

$$
\xi_{1}-\xi_{0}=\int \frac{\mathrm{d} \Phi}{\Phi^{2}}=-\frac{1}{\Phi}
$$

Then, we can obtain a rational function solution:

$$
\Phi\left(\xi_{1}\right)=-\frac{1}{\xi_{1}-\xi_{0}} .
$$

Therefore, the solutions of equation (16) can be shown as

$$
u_{2}(\xi)=\mp a^{-1 / 4} \frac{1}{a^{-1 / 4} \xi-\xi_{0}}-\frac{a_{3}}{4 a_{4}} .
$$

For example, when $k=1, p=2, \omega=2, q=0, r=-12$, $c_{0}=0, c_{1}=0$, and $\xi_{0}=0$, we can obtain a rational function solution of equation (8) as

$$
u_{2}(t, x)=\frac{1}{x-\left(2 t^{\alpha} / \alpha\right)} \text {. }
$$

Case 3. $D_{2}>0, D_{3}=0, D_{4}=0$, and $E_{2}>0 . G(\Phi)$ has two real roots of multiplicities two, namely,

$$
G(\Phi)=(\Phi-\beta)^{2}(\Phi-\gamma)^{2},
$$

where $\beta>\gamma$. By using equation (16), we can obtain

$$
\pm\left(\xi_{1}-\xi_{0}\right)=\int \frac{\mathrm{d} \Phi}{(\Phi-\beta)(\Phi-\gamma)}=\frac{1}{\beta-\gamma} \ln \left|\frac{\Phi-\beta}{\Phi-\gamma}\right| .
$$

When $\Phi>\beta$ or $\Phi<\gamma$, we can obtain the solution of equation (16) as follows:

$$
\begin{aligned}
\Phi\left(\xi_{1}\right) & =\frac{\gamma-\beta}{e^{(\beta-\gamma)\left(\xi_{1}-\xi_{0}\right)}-1}+\gamma \\
& =\frac{\gamma-\beta}{2}\left[\operatorname{coth} \frac{(\beta-\gamma)\left(\xi_{1}-\xi_{0}\right)}{2}-1\right]+\gamma .
\end{aligned}
$$

Then, we have

$$
\begin{aligned}
u_{3}(\xi)= & \frac{(\gamma-\beta) a_{4}^{-1 / 4}}{2}\left[\operatorname{coth} \frac{(\beta-\gamma)\left(a_{4}^{1 / 4} \xi-\xi_{0}\right)}{2}-1\right] \\
& +\gamma-\frac{a_{3}}{4 a_{4}} .
\end{aligned}
$$

When $\gamma<\Phi<\beta$, we can gain the solution of equation (16) as follows: 


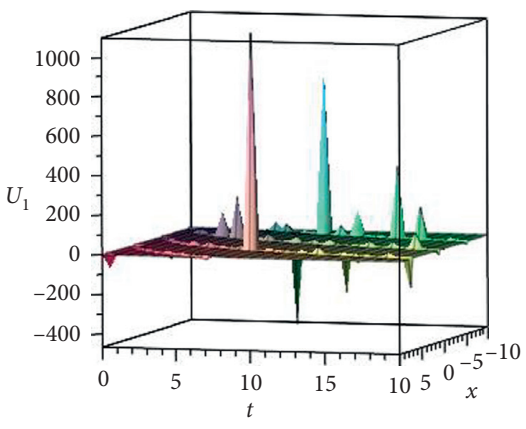

(a)

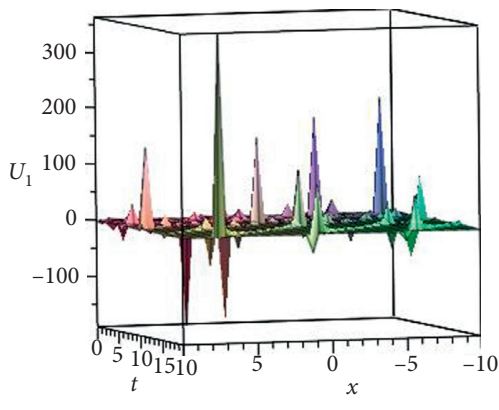

(b)

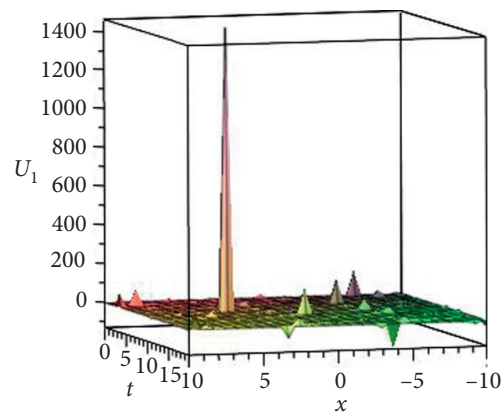

(c)

Figure 1: The graphics of solutions of $u_{1}(t, x)$ for differential values of fractional parameter $\alpha$.

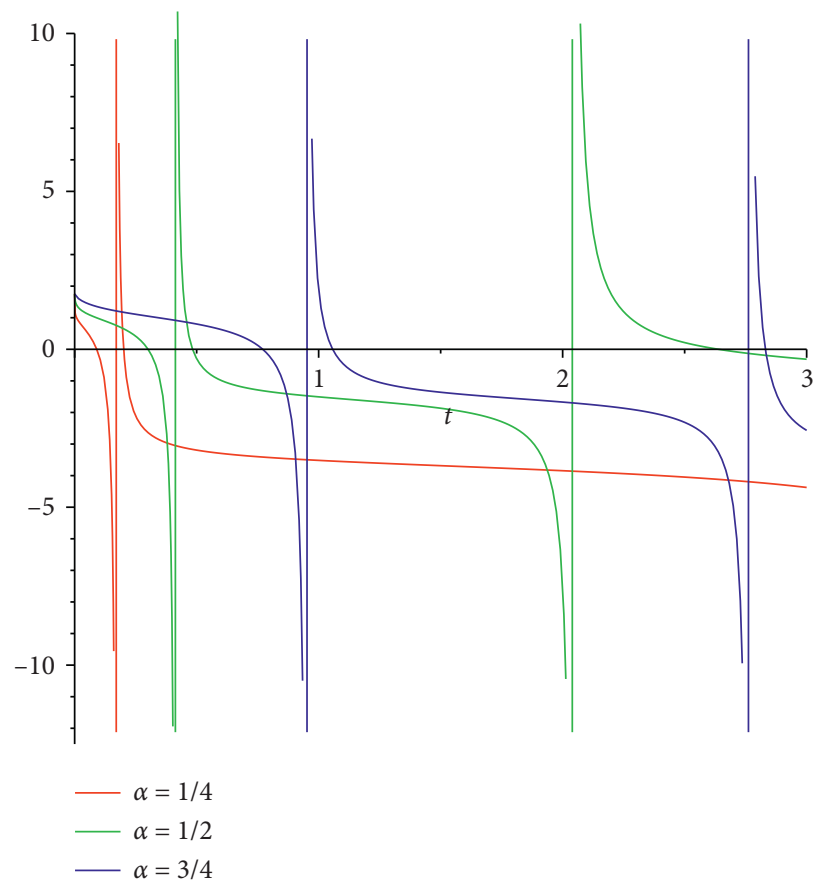

Figure 2: Two-dimensional graphic of solution of $u_{1}(t, x)$ for differential values of fractional parameter $\alpha$.

$$
\begin{aligned}
\Phi\left(\xi_{1}\right) & =\frac{\gamma-\beta}{-e^{(\beta-\gamma)\left(\xi_{1}-\xi_{0}\right)}-1}+\gamma \\
& =\frac{\gamma-\beta}{2}\left[\tan h \frac{(\beta-\gamma)\left(\xi_{1}-\xi_{0}\right)}{2}-1\right]+\gamma,
\end{aligned}
$$

Similarly, we can have

$$
\begin{aligned}
u_{4}(\xi)= & \frac{(\gamma-\beta) a_{4}^{-1 / 4}}{2}\left[\tan h \frac{(\beta-\gamma)\left(a_{4}^{1 / 4} \xi-\xi_{0}\right)}{2}-1\right] \\
& +\gamma-\frac{a_{3}}{4 a_{4}} .
\end{aligned}
$$

We can see that equations (31) and (33) are two solitary wave solutions. Especially, when $k=1, p=4, \omega=2$, $q=-12, r=-12, c_{0}=-2, c_{1}=11 / 16$, and $\xi_{0}=0$, we have $b_{1}=-1, b_{2}=0, b_{3}=1 / 4$, and $-1<\Phi<1$, and then we can obtain a hyperbolic solution of equation (8) as follows. Under the given parameters, we draw the traveling wave $3 \mathrm{D}$ solution surface and corresponding 2D solution graphs for the obtained solution $u_{4}(t, x)$ in Figure 3:

$$
u_{4}(t, x)=\tanh \left(x-\frac{2 t^{\alpha}}{\alpha}\right)-\frac{1}{2} \text {. }
$$

Case 4. $D_{2}>0, D_{3}>0$, and $D_{4}=0 . G(\Phi)$ has two real roots and real roots with multiplicities two, namely,

$$
G(\Phi)=\left(\Phi-\beta_{1}\right)^{2}\left(\Phi-\beta_{2}\right)\left(\Phi-\beta_{3}\right),
$$

where $\beta_{i}(i=1,2,3)$ are real numbers and $\beta_{2}>\beta_{3}$. 


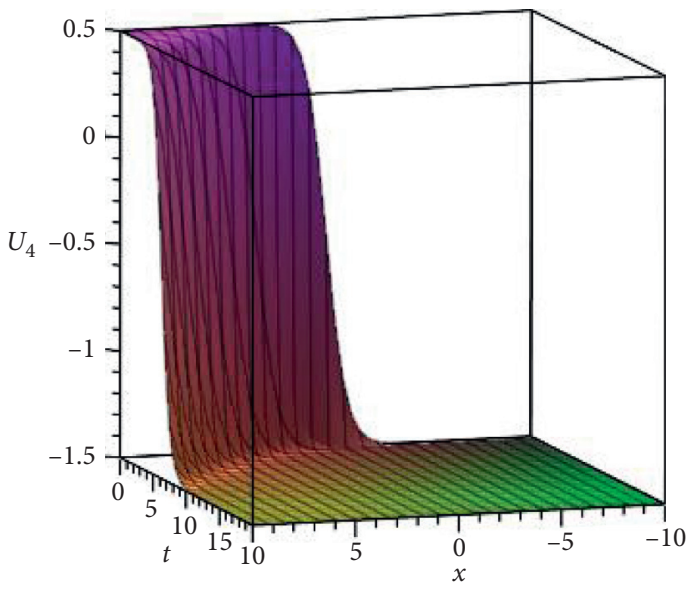

(a)

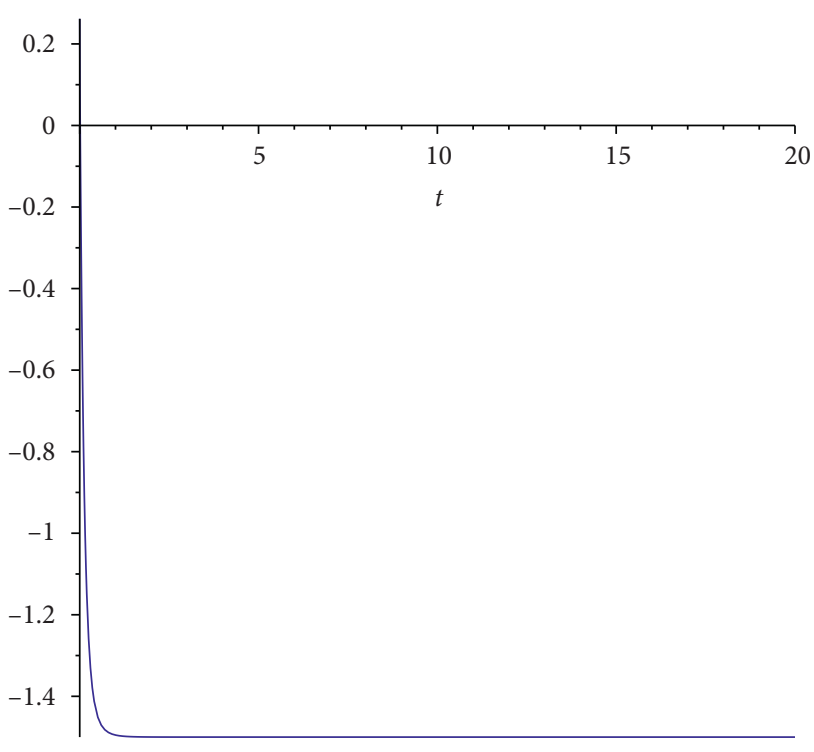

(b)

FIgURE 3: The graphics of solutions of $u_{4}(t, x)$.

When $\beta_{1}>\beta_{2}$ and $\Phi<\beta_{2}$ or when $\beta_{1}<\beta_{3}$ and $\Phi<\beta_{3}$, the following formula can be obtained:

$$
\begin{aligned}
& \pm\left(\xi_{1}-\xi_{0}\right)=\int \frac{\mathrm{d} \Phi}{\sqrt{\left(\Phi-\beta_{1}\right)^{2}\left(\Phi-\beta_{2}\right)\left(\Phi-\beta_{3}\right)}} \\
&=\frac{1}{\left(\beta_{1}-\beta_{2}\right)\left(\beta_{1}-\beta_{3}\right)} \\
& \ln \frac{\left[\sqrt{\left(\Phi-\beta_{2}\right)\left(\beta_{1}-\beta_{3}\right)}-\sqrt{\left(\beta_{1}-\beta_{2}\right)\left(\Phi-\beta_{3}\right)}\right]^{2}}{\left|\Phi-\beta_{1}\right|} .
\end{aligned}
$$

When $\beta_{1}>\beta_{2}$ and $\Phi<\beta_{3}$ or when $\beta_{1}<\beta_{3}$ and $\Phi<\beta_{2}$, we can obtain the solution of equation (28):

$$
\begin{aligned}
\pm\left(\xi_{1}-\xi_{0}\right) & =\int \frac{\mathrm{d} \Phi}{\sqrt{\left(\Phi-\beta_{1}\right)^{2}\left(\Phi-\beta_{2}\right)\left(\Phi-\beta_{3}\right)}} \\
& =\frac{1}{\left(\beta_{1}-\beta_{2}\right)\left(\beta_{1}-\beta_{3}\right)} \ln \frac{\left[\sqrt{\left(\Phi-\beta_{2}\right)\left(\beta_{3}-\beta_{1}\right)}-\sqrt{\left(\beta_{2}-\beta_{1}\right)\left(\Phi-\beta_{3}\right)}\right]^{2}}{\left|\Phi-\beta_{1}\right|} .
\end{aligned}
$$
(16):

When $\beta_{2}>\beta_{1}>\beta_{3}$, we can have the solution of equation

$$
\begin{aligned}
\pm\left(\xi_{1}-\xi_{0}\right) & =\int \frac{\mathrm{d} \Phi}{\sqrt{\left(\Phi-\beta_{1}\right)^{2}\left(\Phi-\beta_{2}\right)\left(\Phi-\beta_{3}\right)}} \\
& =\frac{1}{\left(\beta_{2}-\beta_{1}\right)\left(\beta_{1}-\beta_{3}\right)} \arcsin \frac{\left(\Phi-\beta_{2}\right)\left(\beta_{1}-\beta_{3}\right)+\left(\beta_{1}-\beta_{2}\right)\left(\Phi-\beta_{3}\right)}{\left|\left(\Phi-\beta_{1}\right)\left(\beta_{2}-\beta_{3}\right)\right|} .
\end{aligned}
$$


Especially, when $k=1, p=4, \omega=2, q=-12, r=-12$, $c_{0}=-2, c_{1}=-9 / 16$, and $\xi_{0}=0$, we have $b_{1}=-1, b_{2}=0$, and $b_{3}=0$. By taking them into (38), then we can obtain

$$
u_{5}(t, x)=\sin \left(x-\frac{2 t^{\alpha}}{\alpha}\right)-\frac{1}{2} .
$$

Case 5. $D_{2}>0, D_{3}=0, D_{4}=0$, and $E_{2}=0 . G(\Phi)$ has real roots of multiplicities three and real roots with multiplicities one, namely,

$$
G(\Phi)=(\Phi-\beta)^{3}(\Phi-\gamma)
$$

where $\beta$ and $\gamma$ are real numbers. Using formula (16) can yield

$$
\pm\left(\xi_{1}-\xi_{0}\right)=\int \frac{\mathrm{d} \Phi}{\sqrt{(\Phi-\beta)^{3}(\Phi-\gamma)}}=\frac{2}{\gamma-\beta} \sqrt{\frac{\Phi-\gamma}{\Phi-\beta}} .
$$

When $\Phi>\beta$ and $\Phi>\gamma$ or when $\Phi<\beta$ and $\Phi<\gamma$, the solution of equation (16) is given by

$$
\Phi\left(\xi_{1}\right)=\frac{4(\beta-\gamma)}{(\gamma-\beta)^{2}\left(\xi_{1}-\xi_{0}\right)^{2}-4}+\beta .
$$

Then, we can obtain a rational solution of equation (8) as

$$
u_{6}(\xi)=\frac{4 a^{-1 / 4}(\beta-\gamma)}{\left(a^{1 / 4} \xi-\xi_{0}\right)^{2}(\gamma-\beta)^{2}-4}+\beta-\frac{a_{3}}{4 a_{4}} .
$$

Case 6. $D_{2} D_{3}<0$ and $D_{4}=0 . G(\Phi)$ has real roots of multiplicities two and a pair of conjugate complex roots, namely,

$$
G(\Phi)=\left(\Phi-\beta_{1}\right)^{2}\left[\left(\Phi-\beta_{2}\right)^{2}+\beta_{3}^{2}\right],
$$

where $\beta_{i}(i=1,2,3)$ are real numbers. By using equation (16), we can obtain

$$
\begin{aligned}
\pm\left(\xi_{1}-\xi_{0}\right) & =\int \frac{\mathrm{d} \Phi}{\left(\Phi-\beta_{1}\right) \sqrt{\left(\Phi-\beta_{2}\right)^{2}+\beta_{3}^{2}}} \\
& =\frac{1}{\sqrt{\left(\beta_{1}-\beta_{2}\right)^{2}+\beta_{3}^{2}}} \ln \left|\frac{c_{1} \Phi+c_{2}-\sqrt{\left(\Phi-\beta_{2}\right)^{2}+\beta_{3}^{2}}}{\Phi-\beta_{1}}\right|
\end{aligned}
$$

where $\quad c_{1}=\beta_{1}-2 \beta_{2} / \sqrt{\left(\beta_{1}-\beta_{2}\right)^{2}+\beta_{3}^{2}} \quad$ and $c_{2}=\sqrt{\left(\beta_{1}-\beta_{2}\right)^{2}+\beta_{3}^{2}}-\left(\beta_{1}\left(\beta_{1}-2 \beta_{2}\right) / \sqrt{\left(\beta_{1}-\beta_{2}\right)^{2}+\beta_{3}^{2}}\right)$.

Thus, we obtain the solution of equation (16):

$$
\Phi\left(\xi_{1}\right)=\frac{\left.\left(e^{ \pm \sqrt{\left(\beta_{1}-\beta_{2}\right)^{2}+\beta_{3}^{2}}\left(\xi_{1}-\xi_{0}\right)}-c_{1}\right)+\sqrt{\left(\beta_{1}-\beta_{2}\right)^{2}+\beta_{3}^{2}}\left(2-c_{1}\right)\right)}{\left(e^{ \pm \sqrt{\left(\beta_{1}-\beta_{2}\right)^{2}+\beta_{3}^{2}}}\left(\xi_{1}-\xi_{0}\right)-c_{1}\right)^{2}-1} .
$$

Hence,

$$
u_{7}(\xi)=\frac{\left[e^{ \pm \sqrt{\left(\beta_{1}-\beta_{2}\right)^{2}+\beta_{3}^{2}}\left(a_{4}^{1 / 4} \xi-\xi_{0}\right)}-c_{1}\right]+\sqrt{\left(\beta_{1}-\beta_{2}\right)^{2}+\beta_{3}^{2}}\left(2-c_{1}\right)}{a_{4}^{1 / 4}\left[\left(e^{ \pm \sqrt{\left(\beta_{1}-\beta_{2}\right)^{2}+\beta_{3}^{2}}\left(a_{4}^{1 / 4} \xi-\xi_{0}\right)}-c_{1}\right)^{2}-1\right]}-\frac{a_{3}}{4 a_{4}},
$$

which is a solitary wave solution.

Case 7. $D_{2}>0, D_{3}>0$, and $D_{4}>0 . G(\Phi)$ has four distinct real roots, namely,

$$
G(\Phi)=\left(\Phi-\beta_{1}\right)\left(\Phi-\beta_{2}\right)\left(\Phi-\beta_{3}\right)\left(\Phi-\beta_{4}\right),
$$

where $\beta_{1}, \beta_{2}, \beta_{3}$, and $\beta_{4}$ are real numbers and $\beta_{1}>\beta_{2}>\beta_{3}>\beta_{4}$.

When $\Phi>\beta_{1}$ or $\Phi<\beta_{4}$, we make the following transformation:

$$
\Phi=\frac{\beta_{2}\left(\beta_{1}-\beta_{4}\right) \sin ^{2} \theta-\beta_{1}\left(\beta_{2}-\beta_{4}\right)}{\left(\beta_{1}-\beta_{4}\right) \sin ^{2} \theta-\left(\beta_{2}-\beta_{4}\right)} .
$$

When $\beta_{3}<\Phi<\beta_{2}$, similarly

$$
\Phi=\frac{\beta_{4}\left(\beta_{2}-\beta_{3}\right) \sin ^{2} \theta-\beta_{3}\left(\beta_{2}-\beta_{4}\right)}{\left(\beta_{2}-\beta_{3}\right) \sin ^{2} \theta-\left(\beta_{2}-\beta_{4}\right)} .
$$

By using equation (16), we obtain

$$
\begin{aligned}
\pm\left(\xi_{1}-\xi_{0}\right) & =\int \frac{\mathrm{d} \Phi}{\sqrt{\left(\Phi-\beta_{1}\right)\left(\Phi-\beta_{2}\right)\left(\Phi-\beta_{3}\right)\left(\Phi-\beta_{4}\right)}} \\
& =\frac{2}{\sqrt{\left(\beta_{1}-\beta_{3}\right)\left(\beta_{2}-\beta_{4}\right)}} \int \frac{\mathrm{d} \theta}{\sqrt{1-m^{2} \sin ^{2} \theta}},
\end{aligned}
$$

where $m^{2}=\left(\beta_{1}-\beta_{4}\right)\left(\beta_{2}-\beta_{3}\right) /\left(\beta_{1}-\beta_{3}\right)\left(\beta_{2}-\beta_{4}\right)$.

From equation (50) and the definition of Jacobian elliptic sine function, we obtain 


$$
\sin \theta=\operatorname{sn}\left(\frac{\sqrt{\left(\beta_{1}-\beta_{3}\right)\left(\beta_{2}-\beta_{4}\right)}}{2}\left(\xi_{1}-\xi_{0}\right), m\right) .
$$

Combining equation (51) with expression (48), we can gain the solutions of equation (16):

$$
\Phi\left(\xi_{1}\right)=\frac{\beta_{2}\left(\beta_{1}-\beta_{4}\right) \operatorname{sn}^{2}\left(\sqrt{\left(\beta_{1}-\beta_{3}\right)\left(\beta_{2}-\beta_{4}\right)} / 2\left(\xi_{1}-\xi_{0}\right), m\right)-\beta_{1}\left(\beta_{2}-\beta_{4}\right)}{\left(\beta_{1}-\beta_{4}\right) \operatorname{sn}^{2}\left(\sqrt{\left(\beta_{1}-\beta_{3}\right)\left(\beta_{2}-\beta_{4}\right)} / 2\left(\xi_{1}-\xi_{0}\right), m\right)-\left(\beta_{2}-\beta_{4}\right)},
$$

and then we can give the solution of equation (8):

$$
u_{8}(\xi)=\frac{\beta_{2}\left(\beta_{1}-\beta_{4}\right) a_{4}^{-1 / 4} \operatorname{sn}^{2}\left(\sqrt{\left(\beta_{1}-\beta_{3}\right)\left(\beta_{2}-\beta_{4}\right)} / 2\left(a_{4}^{1 / 4} \xi-\xi_{0}\right), m\right)-\beta_{1}\left(\beta_{2}-\beta_{4}\right)}{\left(\beta_{1}-\beta_{4}\right) \operatorname{sn}^{2}\left(\sqrt{\left(\beta_{1}-\beta_{3}\right)\left(\beta_{2}-\beta_{4}\right)} / 2\left(a_{4}^{1 / 4} \xi-\xi_{0}\right), m\right)-\left(\beta_{2}-\beta_{4}\right)}-\frac{a_{3}}{4 a_{4}} .
$$

Similarly, combining equation (51) with expression (49), we can obtain the solution of equation (8):

$$
u_{9}(\xi)=\frac{\beta_{4}\left(\beta_{2}-\beta_{3}\right) a_{4}^{-1 / 4} s n^{2}\left(\sqrt{\left(\beta_{1}-\beta_{3}\right)\left(\beta_{2}-\beta_{4}\right)} / 2\left(a_{4}^{1 / 4} \xi-\xi_{0}\right), m\right)-\beta_{3}\left(\beta_{2}-\beta_{4}\right)}{\left(\beta_{2}-\beta_{3}\right) \operatorname{sn}^{2}\left(\sqrt{\left(\beta_{1}-\beta_{3}\right)\left(\beta_{2}-\beta_{4}\right)} / 2\left(a_{4}^{1 / 4} \xi-\xi_{0}\right), m\right)-\left(\beta_{2}-\beta_{4}\right)}-\frac{a_{3}}{4 a_{4}} .
$$

Case 8. $D_{2} D_{3} \geq 0$ and $D_{4}<0 . G(\Phi)$ has two different real roots and a pair of conjugate complex roots, namely,

$$
G(\Phi)=\left(\Phi-\beta_{1}\right)\left(\Phi-\beta_{2}\right)\left[\left(\Phi-\beta_{3}\right)^{2}+\beta_{4}^{2}\right],
$$

where $\beta_{1}, \beta_{2}, \beta_{3}$, and $\beta_{4}$ are real constants, $\beta_{1}>\beta_{2}$, and $\beta_{4}>0$.

Making the following transformation, we obtain

$$
\Phi=\frac{c_{1} \cos \theta+c_{2}}{c_{2} \cos \theta+c_{4}}
$$

where $c_{1}=1 / 2\left(\beta_{1}+\beta_{2}\right) c_{3}-1 / 2\left(\beta_{1}-\beta_{2}\right) c_{4}, \quad c_{2}=1 / 2\left(\beta_{1}+\right.$ $\left.\beta_{2}\right) c_{4}-1 / 2\left(\beta_{1}-\beta_{2}\right) c_{3}, \quad c_{3}=\beta_{1}-\beta_{3}-\beta_{4} / m_{1}, c_{4}=\beta_{1}-\beta_{3}-$ $\beta_{4} m_{1}, \quad E=\beta_{4}^{2}+\left(\beta_{1}-\beta_{3}\right)\left(\beta_{2}-\beta_{3}\right) / \beta_{4}\left(\beta_{1}-\beta_{2}\right), \quad$ and $m_{1}=E \pm \sqrt{E^{2}+1}$.

By using equation (16), we obtain:

$$
\begin{aligned}
\xi_{1}-\xi_{0} & =\int \frac{\mathrm{d} \Phi}{\sqrt{ \pm\left(\Phi-\beta_{1}\right)\left(\Phi-\beta_{2}\right)\left(\left(\Phi-\beta_{3}\right)^{2}+\beta_{4}^{2}\right)}} \\
& =\frac{2 m_{1} m_{2}}{\sqrt{\left(\mp 2 \beta_{4} m_{1}\left(\beta_{1}-\beta_{2}\right)\right)}} \int \frac{\mathrm{d} \theta}{\sqrt{1-m_{2}^{2} \sin ^{2} \theta}},
\end{aligned}
$$

where $m_{2}^{2}=2 / 1+m_{1}^{2}$.

From equation (57) and the definition of Jacobian elliptic function, we obtain

$$
\cos \theta=c n\left(\frac{\sqrt{\mp 2 \beta_{4} m_{1}\left(\beta_{1}-\beta_{2}\right)}}{2 m_{1} m_{2}}\left(\xi_{1}-\xi_{0}\right), m_{2}\right) .
$$

Combining equation (58) with expression (56), we can gain the solutions of equation (16):

$$
\Phi\left(\xi_{1}\right)=\frac{c_{1} c n\left(\sqrt{\mp 2 \beta_{4} m_{1}\left(\beta_{1}-\beta_{2}\right)} / 2 m_{1} m_{2}\left(\xi_{1}-\xi_{0}\right), m_{2}\right)+c_{2}}{c_{3} c n\left(\sqrt{\mp 2 \beta_{4} m_{1}\left(\beta_{1}-\beta_{2}\right)} / 2 m_{1} m_{2}\left(\xi_{1}-\xi_{0}\right), m_{2}\right)+c_{3}}
$$


and then we can give the solution of equation (8):

$$
u_{10}(\xi)=\frac{a_{4}^{-1 / 4}\left[c_{1} c n\left(\sqrt{\mp 2 \beta_{4} m_{1}\left(\beta_{1}-\beta_{2}\right)} / 2 m_{1} m_{2}\left(a_{4}^{1 / 4} \xi-\xi_{0}\right), m_{2}\right)+c_{2}\right]}{c_{3} c n\left(\sqrt{\mp 2 \beta_{4} m_{1}\left(\beta_{1}-\beta_{2}\right)} / 2 m_{1} m_{2}\left(a_{4}^{1 / 4} \xi-\xi_{0}\right), m_{2}\right)+c_{3}},
$$

which is an elliptic double periodic function solution.

Case 9. $D_{2} D_{3} \leq 0$ and $D_{4}>0 . G(\Phi)$ has two pairs of conjugate complex roots, namely,

$$
G(\Phi)=\left[\left(\Phi-\beta_{1}\right)^{2}+l_{2}^{2}\right]\left[\left(\Phi-\beta_{2}\right)^{2}+l_{2}^{2}\right]
$$

where $\beta_{1}, \beta_{2}, l_{1}$, and $l_{2}$ are real constants and $l_{1} \geq l_{2}>0$.

Making the following transformation, we obtain

$$
\Phi=\frac{c_{1} \tan \theta+c_{2}}{c_{2} \tan \theta+c_{4}}
$$

where $c_{1}=\beta_{1} c_{3}+l_{1} c_{4}, \quad c_{2}=\beta_{1} c_{4}-l_{1} c_{3}, \quad c_{3}=-l_{1}-l_{2} / m_{1}$, $c_{4}=\beta_{1}-\beta_{2}, \quad E=\left(\beta_{1}-\beta_{2}\right)^{2}+l_{1}^{2}+l_{2}^{2} / 2 l_{1} l_{2}, \quad$ and $m_{1}=E+$ $\sqrt{E^{2}-1}$.

By using equation (16), we obtain

$$
\begin{aligned}
\xi_{1}-\xi_{0} & =\int \frac{\mathrm{d} \Phi}{\sqrt{\left[\left(\Phi-\beta_{1}\right)^{2}+l_{2}^{2}\right]\left[\left(\Phi-\beta_{2}\right)^{2}+l_{2}^{2}\right]}} \\
& =\frac{c_{3}^{2}+c_{4}^{2}}{l_{2} \sqrt{\left(c_{3}^{2}+c_{4}^{2}\right)\left(m_{1}^{2} c_{3}^{2}+c_{4}^{2}\right)}} \int \frac{\mathrm{d} \theta}{\sqrt{1-m_{2}^{2} \sin ^{2} \theta}},
\end{aligned}
$$

where $m_{2}^{2}=m_{1}^{2}-1 / m_{1}^{2}$.

From equation (62) and the definition of Jacobian elliptic function, we obtain

$$
\sin \theta=\operatorname{sn}\left(\frac{l_{2} \sqrt{\left(c_{3}^{2}+c_{4}^{2}\right)\left(m_{1}^{2} c_{3}^{2}+c_{4}^{2}\right)}}{c_{3}^{2}+c_{4}^{2}}\left(\xi_{1}-\xi_{0}\right), m_{2}\right),
$$

$$
\cos \theta=c n\left(\frac{l_{2} \sqrt{\left(c_{3}^{2}+c_{4}^{2}\right)\left(m_{1}^{2} c_{3}^{2}+c_{4}^{2}\right)}}{c_{3}^{2}+c_{4}^{2}}\left(\xi_{1}-\xi_{0}\right), m_{2}\right) .
$$

Combining equations (64) and (65) with expression (62), we can gain the solutions of equation (16):

$$
\Phi\left(\xi_{1}\right)=\frac{c_{1} \operatorname{sn}\left(\mu\left(\xi_{1}-\xi_{0}\right), m_{2}\right)+c_{2} c n\left(\mu\left(\xi_{1}-\xi_{0}\right), m_{2}\right)}{c_{3} \operatorname{sn}\left(\mu\left(\xi_{1}-\xi_{0}\right), m_{2}\right)+c_{4} c n\left(\mu\left(\xi_{1}-\xi_{0}\right), m_{2}\right)},
$$

where $\mu=l_{2} \sqrt{\left(c_{3}^{2}+c_{4}^{2}\right)\left(m_{1}^{2} c_{3}^{2}+c_{4}^{2}\right)} / c_{3}^{2}+c_{4}^{2}$. Then, we can give the solution of equation (8):

$$
u_{11}(\xi)=\frac{a_{4}^{-1 / 4} c_{1} \operatorname{sn}\left(\mu\left(a_{4}^{1 / 4} \xi-\xi_{0}\right), m_{2}\right)+a_{4}^{-1 / 4} c_{2} c n\left(\mu\left(a_{4}^{1 / 4} \xi-\xi_{0}\right), m_{2}\right)}{c_{3} \operatorname{sn}\left(\mu\left(a_{4}^{1 / 4} \xi-\xi_{0}\right), m_{2}\right)+c_{4} c n\left(\mu\left(a_{4}^{1 / 4} \xi-\xi_{0}\right), m_{2}\right)}-\frac{a_{3}}{4 a_{4}},
$$

which is an elliptic double periodic function solution.

\section{Conclusion}

By using the complete discrimination system method, we obtain exact traveling wave solutions to generalized fractional Gardner equations under the given parameter conditions. Many exact solutions have been obtained, which include hyperbolic function solutions, Jacobi elliptic function solutions, trigonometric function solutions, and rational function solutions. Compared with the previous work, the solution obtained in the paper has not been reported. Furthermore, the method we employ here can be used to analyze the exact solutions to other FPDEs.

\section{Data Availability}

No data were used to support this study.

\section{Conflicts of Interest}

The authors declare that there are no conflicts of interest regarding the publication of this paper.

\section{Authors' Contributions}

All authors read and approved the final manuscript.

\section{Acknowledgments}

This work was supported by Science Research Fund of Education Department of Sichuan Province of China under grant no. 18ZB0537 and Scientific Research Funds of Chengdu University under grant no. 2081920034.

\section{References}

[1] D. Baleanu, M. Inc, A. Yusuf, and A. I. Aliyu, "Lie symmetry analysis, exact solutions and conservation laws for the time fractional Caudrey-Dodd-Gibbon-Sawada-Kotera equation," Communications in Nonlinear Science and Numerical Simulation, vol. 59, pp. 222-234, 2018.

[2] D. Baleanu and A. Fernandez, "On some new properties of fractional derivatives with Mittag-Leffler kernel," Communications in Nonlinear Science and Numerical Simulation, vol. 59, pp. 444-462, 2018. 
[3] W. Rui, "Applications of homogenous balanced principle on investigating exact solutions to a series of time fractional nonlinear PDEs," Communications in Nonlinear Science and Numerical Simulation, vol. 47, pp. 253-266, 2017.

[4] D. Baleanu, M. Jleli, S. Kumar, and B. Samet, "A fractional derivative with two singular kernels and application to a heat conduction problem," Advance in Difference Equations, vol. 2020, 2020.

[5] D. Kumar, A. R. Seadawy, and A. K. Joardar, "Modified Kudryashov method via new exact solutions for some conformable fractional differential equations arising in mathematical biology," Chinese Journal of Physics, vol. 56, no. 1, pp. 78-85, 2018.

[6] D. Kumar and M. Kaplan, "New analytical solutions of (2+1)dimensional conformable time fractional Zoomeron equation via two distinct techniques," Chinese Journal of Physics, vol. 56, no. 5, pp. 2173-2185, 2018.

[7] S. S. Ray, "Analytical solution for the space fractional diffusion equation by two-step Adomian decomposition method," Communications in Nonlinear Science and Numerical Simulation, vol. 14, pp. 1295-1306, 2009.

[8] R. Sahadevan and P. Prakash, "Exact solution of certain time fractional nonlinear partial differential equations," Nonlinear Dynamics, vol. 86, pp. 1-15, 2016.

[9] W. Rui, "Applications of integral bifurcation method together with homogeneous balanced principle on investigating exact solutions of time fractional nonlinear PDEs," Nonlinear Dynamics, vol. 91, no. 1, pp. 697-712, 2018.

[10] I. Aliyu, M. Inc, A. Yusuf, and D. Baleanu, "Adomian-Padé approximate solutions to the conformable nonlinear heat transfer equation," Thermal Science, vol. 23, no. 1, pp. 235-242, 2019.

[11] D. Baleanu, M. Inc, A. I. Aliyu, and A. Yusuf, "The investigation of soliton solutions and conservation laws to the coupled generalized Schrödinger-Boussinesq system," Waves in Random and Complex Media, vol. 29, no. 1, pp. 77-92, 2019.

[12] A. I. Aliyu, F. Tchier, M. Inc, A. Yusuf, and D. Baleanu, "Dynamics of optical solitons, multipliers and conservation laws to the nonlinear Schrödinger equation in $(2+1)$-dimensions with non-Kerr law nonlinearity," Journal of Modern Optics, vol. 66, no. 2, pp. 136-142, 2019.

[13] M. Inc, H. I. Abdel Gawad, M. Tantawy, and A. Yusuf, "On multiple soliton similariton-pair solutions, conservation laws via multiplier and stability analysis for the Whitham-BroerKaup equations in weakly dispersive media," Mathematical Methods in the Applied Sciences, vol. 42, no. 7, pp. 2455-2464, 2019.

[14] E. C. Aslan and M. Inc, "Optical soliton solutions of the NLSE with quadratic-cubic-Hamiltonian perturbations and modulation instability analysis," Optik, vol. 196, Article ID 162661, 2019.

[15] Z. Korpinar, M. Inc, M. Bayram, and M. S. Hashemi, "New optical solitons for Biswas-Arshed equation with higher order dispersions and full nonlinearity," Optik.vol. 206, Article ID 163332, 2020.

[16] S. Kumar and D. Baleanu, "A new numerical method for time fractional non-linear sharma-tasso-oliver equation and kleinGordon equation with exponential kernel law," Frontiers in Physics, vol. 8, p. 136, 2020.

[17] Z. Korpinar, F. Tchier, and M. Inc, "On optical solitons of the fractional (3+1)-dimensional NLSE with conformable derivatives," Frontiers in Physics, vol. 8, p. 87, 2020.

[18] T. Korpinar, Z. Korpinar, M. Inc, and D. Baleanu, "Geometric phase for timelike spherical normal magnetic charged particles optical ferromagnetic model," Journal of Taibah University for Science, vol. 14, no. 1, pp. 742-749, 2020.

[19] H. Rezazadeh, A. Korkmaz, M. Eslami, and S. M. MirhosseiniAlizamini, "A large family of optical solutions to KunduEckhasus model by a new auxiliary equation method," Optical and Quantum Electronics, vol. 51, p. 84, 2019.

[20] N. Raza, U. Afzal, A. R. Butt et al., "A large family of optical solutions to Kundu-Eckhasus model by a new auxiliary equation method," Optical and Quantum Electronics, vol. 51, p. 84, 2019 .

[21] W. Gao, H. Rezazadeh, Z. Pinar, H. M. Baskonus, S. Sarwar, and G. Yel, "Novel explicit solutions for the nonlinear Zoomeron equation by using newly extended direct algebraic technique," Optical and Quantum Electronics, vol. 52, p. 52, 2019.

[22] N. Savaissou, B. Gambo, H. Rezazadeh, A. Bekir, and S. Y. Doka, "Exact optical solitons to the perturbed nonlinear Schrödinger equation with dual-power law of nonlinarity," Optical and Quantum Electronics, vol. 52, p. 318, 2020.

[23] C. Park, M. M. A. Khater, A.-H. Abdel-Aty et al., "Dynamical analysis of the nonlinear complex fractional emerging telecommunication model with higher-order dispersive cubicquintic," Alexandria Engineering Journal, vol. 59, no. 3, pp. 1425-1433, 2020.

[24] H. Rezazadeh, J. Vahidi, A. Zafar, and A. Bekir, "The functional variable method to find new exact solutions of the nonlinear evolution equations with Dual-Power-Law nonlinearity," International Journal of Nonlinear Sciences and Numerical Simulation, vol. 3-4, pp. 249-257, 2019.

[25] O. Guner, H. Atik, and A. A. Kayyrzhanovich, "New exact solution for space-time fractional differential equations via (G'/G)-expansion method," Optik, vol. 130, pp. 696-701, 2017.

[26] M. Ekici, "Soliton and other solutions of nonlinear time fractional parabolic equations using extended $\mathrm{G}^{\prime} / \mathrm{G}$-expansion method," Optik, vol. 130, pp. 1312-1319, 2017.

[27] Z. S. Wen, "The generalized bifurcation method for deriving exact solutions of nonlinear space-time fractional partial differential equations," Applied Mathematics and Computation, vol. 366, Article ID 124735, 2020.

[28] A. Das, N. Ghosh, and K. Ansari, "Bifurcation and exact traveling wave solutions for dual power Zakharov-KuznetsovBurgers equation with fractional temporal evolution," Computers \& Mathematics with Applications, vol. 75, no. 1, pp. 59-69, 2018.

[29] A. R. Adem, "Symbolic computation on exact solutions of a coupled Kadomtsev-Petviashvili equation: Lie symmetry analysis and extended tanh method," Computers \& Mathematics with Applications, vol. 74, no. 8, pp. 1897-1902, 2017.

[30] Z. Y. Zhang and G. F. Li, "Lie symmetry analysis and exact solutions of the time-fractional biological population model," Physica A: Statistical Mechanics and Its Applications, vol. 540, Article ID 123134, 2020.

[31] B. Lu, "The first integral method for some time fractional differential equations," Journal of Mathematical Analysis and Applications, vol. 395, no. 2, pp. 684-693, 2012.

[32] M. Odabasi, "Traveling wave solutions of conformable timefractional Zakharov-Kuznetsov and Zoomeron equations," Chinese Journal of Physics, vol. 64, pp. 194-202, 2020.

[33] O. Guner and H. Atik, "Soliton solution of fractional-order nonlinear differential equations based on the exp-function method," Optik, vol. 127, no. 20, Article ID 10076, 2016.

[34] Y. Pandir and H. H. Duzgun, "New exact solutions of time fractional Gardner equation by using new version of 
F-expansion method," Communications in Theoretical Physics, vol. 67, no. 1, pp. 9-14, 2017.

[35] M. Eslami, "Exact traveling wave solutions to the fractional coupled nonlinear Schrodinger equations," Applied Mathematics and Computation, vol. 285, pp. 141-148, 2016.

[36] H. Rezazadeh, D. Kumar, T. A. Sulaiman, and H. Bulut, "New complex hyperbolic and trigonometric solutions for the generalized conformable fractional Gardner equation," Modern Physics Letters B, vol. 33, no. 17, Article ID 1950196, 2019.

[37] C. S. Liu, "Exact travelling wave solutions for (1+1)-dimensional dispersive long wave equation," Chinese Physics, vol. 14, pp. 1710-1715, 2005.

[38] C. Wu and W. Rui, "Method of separation variables combined with homogenous balanced principle for searching exact solutions of nonlinear time-fractional biological population model," Communications in Nonlinear Science and Numerical Simulation, vol. 63, pp. 88-100, 2018.

[39] W. Rui, "Idea of invariant subspace combined with elementary integral method for investigating exact solutions of time-fractional NPDEs," Applied Mathematics and Computation, vol. 339, pp. 158-171, 2018.

[40] R. Khalil, M. Al Horani, A. Yousef, and M. Sababheh, "A new definition of fractional derivative," Journal of Computational and Applied Mathematics, vol. 264, pp. 65-70, 2014.

[41] D. Cao, "The classification of the single traveling wave solutions to the time-fraction Gardner equation," Chinese Journal of Physics, vol. 59, pp. 379-392, 2019.

[42] N. Raza, "Exact periodic and explicit solutions of the conformable time fractional Ginzburg Landau equation," Optical and Quantum Electronics, vol. 50, pp. 154-170, 2018.

[43] H. Rezazadeh, H. Tariq, M. Eslami, M. Mirzazadeh, and Q. Zhou, "New exact solutions of nonlinear conformable time-fractional Phi-4 equation," Chinese Journal of Physics, vol. 56, no. 6, pp. 2805-2816, 2018.

[44] C. Chen and Y.-L. Jiang, "Simplest equation method for some time-fractional partial differential equations with conformable derivative," Computers \& Mathematics with Applications, vol. 75, no. 8, pp. 2978-2988, 2018.

[45] A. Akbulut and M. Kaplan, "Auxiliary equation method for time-fractional differential equations with conformable derivative," Computers \& Mathematics with Applications, vol. 75, no. 3, pp. 876-882, 2018.

[46] Q. Feng, "A new approach for seeking coefficient function solutions of conformable fractional partial differential equations based on the Jacobi elliptic equation," Chinese Journal of Physics, vol. 56, no. 6, pp. 2817-2828, 2018.

[47] E. Ahmet Gökdoğan, "Solution of conformable fractional ordinary differential equations via differential transform method," Optik, vol. 128, pp. 264-273, 2017.

[48] Y. Xie, Z. Yang, and L. Li, "New exact solutions to the high dispersive cubic-quintic nonlinear Schrödinger equation," Physics Letters A, vol. 382, no. 36, pp. 2506-2514, 2018.

[49] Y. Kai, "The classification of the single traveling wave solutions to the variant boussinesq equations," Pramana, vol. 87, pp. 59-63, 2016.

[50] C. S. Liu, "Classification of all single traveling wave solutions to Calogero-Degasperis-Focas equation," Communications in Theoretical Physics, vol. 48, pp. 601-604, 2017. 